\title{
REACTIVE OPERATING THEATRE SCHEDULING
}

\author{
BELINDA SPRATT
}

(Received 4 June 2018; first published online 15 August 2018)

2010 Mathematics subject classification: primary 90B36; secondary 68T20.

Keywords and phrases: operating theatre planning and scheduling, mixed integer nonlinear programming, metaheuristics, reactive, robust.

The operating theatre (OT) department is often described as a hospital's engine. Depending on the hospital, the operating rooms (ORs) often account for the majority of patient flow and can be one of the largest sources of hospital costs and revenue. Inefficient use of the ORs and related resources can result in increased costs and a decrease in patient and staff welfare. The OT schedules implemented by hospitals can have a large impact on patient health. The scheduling of surgeries is a complex process, yet some surgical departments rely on simple heuristics and general intuition to produce daily or weekly OT schedules.

Throughout this thesis, a number of mathematical techniques are implemented to schedule surgical procedures with the aim of reducing elective surgery waiting lists and improving patient health. Three problems are addressed, based on a case study of a large Australian public hospital: an OT planning problem [1], a dynamic OT planning problem and a real-time reactive OT scheduling problem. To ensure the models used are realistic, consultant availability, OR type, stochastic surgical durations, nonelective arrivals and waiting list management policies are incorporated.

In solving the static and dynamic OT planning problems, a number of innovative hyper and hybrid metaheuristic techniques are presented to ensure that feasible solutions are provided in short amounts of computation time. Given the intensive computational efficiency requirements of OT scheduling problems, two constructive heuristics and a reactive rescheduling framework are presented.

The work presented here can be used to vastly improve the scheduling system of surgical departments by reducing the number of cancellations, decreasing expected overtime and improving patient health. A number of interactive strategies are provided that can be implemented in the case study hospital to increase OT utilisation and reduce elective surgery waiting time.

Thesis submitted to Queensland University of Technology in December 2017; degree approved 21 March 2018; principal supervisor Erhan Kozan, associate supervisor Glen Tian.

(c) 2018 Australian Mathematical Publishing Association Inc. 


\section{Reference}

[1] B. Spratt and E. Kozan, 'Waiting list management through master surgical schedules: a case study', Oper. Res. Health Care 10 (2016), 49-64.

BELINDA SPRATT, School of Mathematical Sciences, Queensland University of Technology, 2 George St, Brisbane 4000, Australia

e-mail: b.spratt@qut.edu.au 